\title{
Mental Health and Social Support Impact on Gender Inequality: A Psychosocial Perspective and Implications
}

\author{
Purushothaman $\mathrm{T}^{1}$, Mangayarkarasi ${ }^{2}$ \\ ${ }^{1}$ Scientist D, DRDO, SCC SI, Lines Bhopal. \\ ${ }^{2}$ Assistant Professor, Department of Women Studies, Bharathiar University Coimbatore \\ Corresponding author: Purushothaman $\mathrm{T}$ \\ Email - tp_man@rediff.com
}

\begin{abstract}
Gender difference is a broad area of study which needs much exploration. From biological to physiological, psychological to social front, differences are bound to be, without which humans cease to exist. The significance lies where and to what extent the difference exists on the continuum. Based on supporting reviews this paper attempts to study mental health and social support on individuals among genders. With reviews and data from appropriate tools on a sample of 471 (232 Males and 238 Females) ANOVA revealed the differences among the genders. Significant effects of gender are evident on both the variables with insignificant interaction effects along with age. The implications are discussed on the psychological and social front.
\end{abstract}

Keywords: gender, mental health, social support.

(Paper received $-7^{\text {th }}$ January 2018, Peer review completed $-18^{\text {th }}$ February 2018)

(Accepted $-25^{\text {th }}$ February 2018)

\section{INTRODUCTION}

When we study about individuals, an obvious question which creeps is what are differences among individuals? A lot of issues have to be dealt with before answering the question. Gender is a prime area of concern while looking at individuals. Mainstreaming gender within institutions has remained superficial. National health policies and programmes that have gender integrally woven into their objectives and activities are less in number. Researches that generate gender-specific data, in health and training, have received scarce attention.

At a more practical level, the goal of reducing inequality among individuals should be of improving holistic health of individuals and communities. Policies and schemes resources that ensure girls to attend school and programmes to improve quality of life could be considered as example for former while those that improve parenting skills and encourage schools to prevent bullying for latter.

There are vivid examples where gender as a variable has implications. Failing to capture gender differences leads to omissions in complexity and errors in understanding that can lead to poor evidence and inappropriate outcomes [1]. Gender is used as a variable would present a way forward to frame gender concerns of inequality within the context of caste, class, ethnicity and other sources of health inequalities; and to work alongside other movements for social justice.

Social support without accounting gender remains a challenge. The empirical evidence supporting how it works is elusive as any nurturance or grooming is just a form of perceived social support. Social relationships and networks can also act as protective factors against the onset or recurrence of mental illhealth and enhance recovery from mental disorders. Interaction with other people is an inevitable 
prerequisite for human development.

Males have better mental health than females, as there are multiple roles, which are bound to impact mental health of females. Presence of depression and distress were more pronounced in females [2]. The multiple roles that women fulfill in society put them at greater risk of experiencing mental disorders than men in the community. Women continue to bear the burden of responsibility associated with being wives, mothers, educators and care taker's of others. While they are increasingly becoming an essential part of the work force, in one-quarter to one-third of households they are the prime source of income too [3]. Studies reveal that women have larger social networks and they give and receive more support than men. Nevertheless, women have higher rates of psychological distress than men [4].

Though mental and behavioral disorders are result of interaction between biological, psychological and social factors, it has been observed that most of the research in psychology has been directed at studying the individual level with minimum attention to the economic, socio-political and cultural, conditions/contexts [5] which are predetermined by gender roles. Psychoanalytic view of Sigmund Freud [6] claims the individual variability of drive strength which further emphasizes that, a constitution determines certain contents of unconscious with some variation. It also postulates the influences of early life experiences (what happens during childhood) leads to circumstances in adult life. In Structural Model of Mental Health [7] the determinants are grouped as individual factors, social support, and cultural values and off course gender is an individual factor in any study.

A meta-analysis of 42 studies, controlling socio- demographic and physical health factors, concluded that active religious involvement increased the odds of being alive by $26 \%$. These effects were stronger for women than men, possibly due to differences between men and women in the psychosocial support that they receive from religion [8]. Social isolation and lack of social support are linked with increased likelihood of heart disease, complications in pregnancy, delivery and suicide [9] hence more prone to contribute for differences among gender. Precisely how these will affect health in the coming years is more difficult to predict, but rapid changes in disease, growing health inequalities and disruption of social cohesion and health sector resilience are to be expected [10]. Inefficient ways of dealing with health problems are thus overpowering more effective, efficient and more equitable [11], ways of organizing health care and improving health [12].

In addition to pressures placed on women because of their expanding and often conflicting roles, they face significant sex discrimination and associated poverty, hunger, malnutrition, overwork with domestic and sexual violence [13]. Violence against women adds to a major social and public health problem, affecting women of all ages, cultural backgrounds and income levels. Traumatic life events are associated with deteriorated mental health more prominent among women [14]. Molestation and physical abuse were significant predictors of psychosis along with serious injury or assault and violence at home. Social support is a broad and complex construct of perceived comfort. Caring or help a person receives from other people or groups make them less vulnerable to stress. It is defined as 'a process through which help is exchanged with others in an attempt to facilitate one or more adaptational goals' [15]. It varies in terms of who provides support and what is provided.

Without social interaction the whole potential for development of the infant remains totally unfulfilled which has been shown by studies of children who have been left without any human contact (wolf children). Some authors regard the availability of social support as directly contributing to increased mental health whereas others see its role mainly as a buffering one in face of stressful adversities or life events [16]. Evidence indicates a greater risk for specific psychiatric disorders, differentially for men and women [17]. Mental health outcomes differ in sexes [18]. A contradictory study also shows that, effect of marital status; social support within and outside the workplace; social networks on subsequent occurrence of psychological distress were similar for men and women [19]. Women have higher mortality rates than men and women in the sub-Saharan region have higher maternal mortality rates than women in both developed and developing countries [20] which reveals cultural effect on health in gender variations.

Female graduates of coeducational institutions are significantly low in adjustment dimensions mainly home, social and emotional than the women-only institutional counterparts [21]. Family is considered the cradle of future society within which adolescents learn who and what they are [22]. The reviews give us enough food for thought regarding the differences among genders on the psychosocial perspective. 


\section{METHODOLOGY}

Are there significant variations of Mental Health, Social Support in relation to Gender and Age among Indians? Is there any relation between Mental Health, Social Support, Age and Gender among the Indian adults? The above questions have led to the following Hypothesis

$\mathbf{H}_{1}$ There will be relationships between Mental Health, Social Support, age and Gender.

$\mathbf{H}_{2}$ There will be effects Gender and age on Mental Health and Social Support.

The aim of the study is analyze mental health and social support in the sample to overview variances among genders.

The objectives of the present study necessitated collecting the data from the adult population. The data was collected from candidates who appeared for the officer's selection as the researcher was part of the assessment team. The candidates appearing for officer's selection range from various age groups from 18 to 45 years. The sample consisted of participants across the country which reasonably avoids cultural biases. The data was collected after their testing hours with willing consent from the participants without disturbing the testing schedule. This age group falls in the later adolescence and early adulthood as well as psycho-socially under a development stage where they undergo a period of heightened sensitivity, identity development, acquisition of life roles, moving towards functional autonomy, along with the gender identity [23]. Further, they represent the majority of the available sample and the particular age group sample is young and energetic.

\section{Sample}

The sample consisted of 471 participants out of whom 232 were males and 238 were females. The average age was 23.81 years. The investigator believed that the sample is typical of the population and hence a good representative of population. Assuming the concerned cases to be typical nature of the population within the age frame using the judgmental sampling (Singh, 2008), the sample was identified as regionwise sample.

The following tools were used for the study -

1. Mental Health Scale (MHS) [24] (60 items with reliability 0.88)

2. Sense of Support Scale (SOSS) [25] (21 items with reliability 0.86).

A specific data sheet was designed for the purpose of study. This data sheet included the questionnaire of Social Support and Mental Health in a sequential order. The variables were not specified to avoid biases as the participants were literate. Prior to administering the test, the participants were asked to fill the demographic details required for the study such as age and gender. The Social Support and Mental Health score was arrived as per the norms of the tool individually and each participant's scores on the measures are sequentially fed in a master data sheet for further analysis.

\section{STATISTICAL ANALYSIS}

ANOVA was used to analyze the variances of Social Support and Mental Health on gender. A factorial $2 \times 2$ design encompassing the participants two age groups \{ages 18-24 [Group I] \& 25-30 [Group II] $\}$ and two genders \{males \& females\} studied separately for each variable Social Support, and Mental Health to see the main effects as well as the interaction effects of the demographic variables on the psychological variables. 


\section{RESULTS}

Table 1 - One way ANOVA between Genders on the various scales

\begin{tabular}{|c|c|c|c|c|c|}
\hline \multirow{2}{*}{ Variables } & \multicolumn{2}{|c|}{ Males } & \multicolumn{2}{c|}{ Females } & \multirow{2}{*}{ F $(1,470)$} \\
\cline { 2 - 5 } & Mean & SD & Mean & SD & \\
\hline Social Support & 45.50 & 7.76 & 48.18 & 6.56 & 18.993 ** \\
\hline Mental Health & 88.19 & 12.70 & 93.31 & 10.90 & $25.641^{* *}$ \\
\hline
\end{tabular}

${ }^{* *} \mathrm{p}<0.01$ level

Table 1 displays the mean score of Social Support among the males was found to be $\mathrm{M}=45.50$ (SD $=7.76$ ) among the males, and $\mathrm{M}=48.18$ ( $\mathrm{SD}=6.56$ ) among the females, being slightly higher than that of the males. The Mental Health was found to be $\mathrm{M}=$ of $88.19(\mathrm{SD}=12.70)$ among the males and 93.31 ( $\mathrm{SD}=$ 10.90) among the females. The mean score of Mental Health of females was found to be more than that of males.

Table 2 - 'F' values of main effects and interaction effects of Age, Gender, on variables ofSocial support, and Mental Health

\begin{tabular}{|c|c|c|c|c|}
\hline \multirow{2}{*}{} & \multirow{2}{*}{ Variables } & \multirow{2}{*}{ Df } & \multicolumn{2}{|c|}{ F Values } \\
\cline { 3 - 5 } & & & SS & MH \\
\hline Main & Gender & 1 & $13.427^{* *}$ & $16.885^{* *}$ \\
\cline { 2 - 5 } Effects & Age & 1 & 1.806 & .094 \\
\hline $\begin{array}{c}\text { Interaction } \\
\text { E effects }\end{array}$ & Gender \&Age & 1 & 1.138 & .009 \\
\hline \multicolumn{2}{|l|}{} & & & \\
\hline
\end{tabular}

Table 2 present the main effects and the interaction effects of the demographic variables on the psychological variables. Main effects were found on the psychological variables of Social Support, and Mental Health by the demographic variable of Gender on sample at the highest significance level of $p<0.01$ and insignificant interaction effects of Gender and Age are seen on the Mental Health and social support.

\section{DISCUSSION}

The results open an opportunity for more exploration. Studies on health outcomes across genders distinguish between vulnerabilities linked to gendered life experience and the dynamic nature of gendered health vulnerabilities. Given that gender-based risks are, in principle, amenable to social change, they offer untapped potential for health interventions [26].

The males and the females in the study differ among themselves on their levels of mental health and as well as social support. The result here supports the hypotheses. The reasons are many. The differences do prevail from birth and without which the world may cease to exist. But this is neither universal nor uniform throughout the lifecycle and is influenced by ethnicity, sexual orientation, socio-economic status, geography and age being other factors [27]. Male, life expectancy is generally lower than that of females due to higher levels of risk taking particularly among younger men, negative health behaviors and lower participation in preventative health measures. Men who embrace the dominant view of masculinity in our society are more vulnerable to health risks. Men's job in factories causes various physiological problems [28]. Women involved in health care [29] are liable for more professional health hazards and infections and more exposed to psycho-social risks [30] and its causative towards mental health [31]. Analysis of gender differences [3] is a multi-dimensional structure with lot of attributes. The figure below shows 
various issues exclusively in gender analysis alone. Gender, a very comprehensive variable, can be studied as a separate entity.

Figure - Gender analysis in health

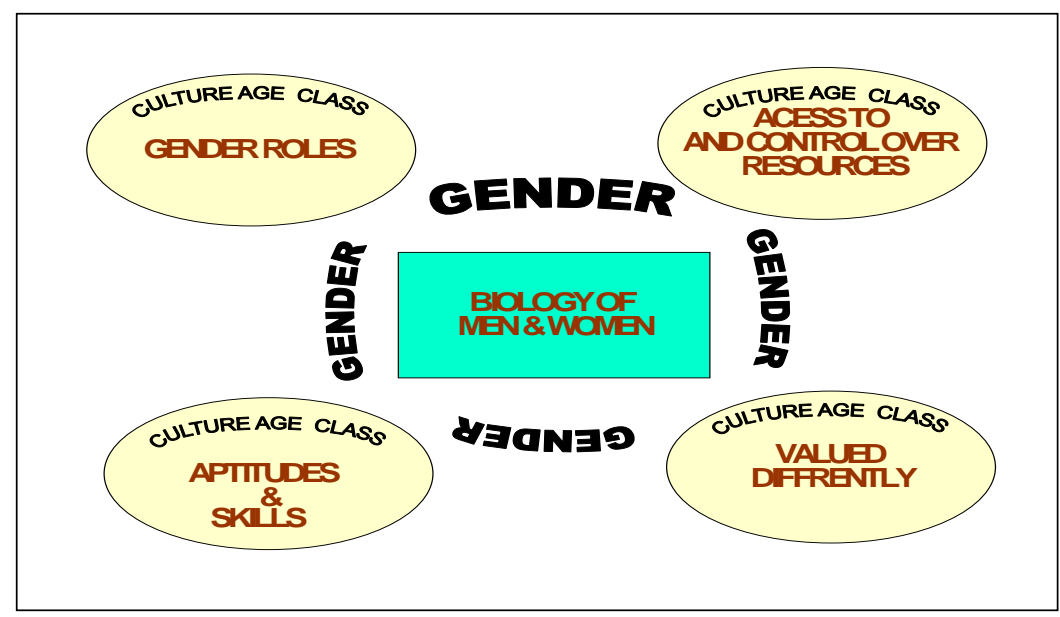

The results reveal the variance of the gender among the variables emphasizing the relevance of the above structure. As long as these shortcomings remain private and individual concern, rather than the collective responsibility of gender, without resolution, health systems will continue to function in a skewed manner, serving to replicate inequalities in the health labour force and in society more broadly [32]. The results reveal higher levels of mental health in women which implies that psychological variables are dynamic in nature. This can be taken as a welcome sign where the transformation of improved mental health has just begun. Another attribute of difference between genders is the intersections between paid and unpaid work. Studies show that while men's blood pressure declines when coming home, women's often rises [33]. Men were more vulnerable to the negative effects of stressors associated with pay and benefits, and women were more vulnerable to the negative outcomes of health and not by differential exposure to them [34]. Gender based risks are often untapped potential in health interventions [26].

Meta-analytic studies on mental health in relation to work environment reveal that high efforts and low rewards are prospective risk factors for common mental disorders and suggest a favorable psycho-social work environment is important for mental health. Also, the impact of work stressors on common mental disorders differs for women and men. Apart from the pressures placed on women because of their expanding and often conflicting roles, they face significant sex discrimination and associated poverty, hunger, malnutrition, overwork and domestic and sexual violence [13]. Therefore, women have been more likely than men to be prescribed psychotropic drugs. Apart from the above, violence against women adds to a major social and public health problem, affecting women of all ages, cultural backgrounds and income levels. Previous studies of the emotional adjustment indicate that it was critically disturbed by gender [35]. In addition to the economic losses that mental disorders represent, intangible costs in terms of human suffering and the total impact of these mental health problems on physical disorders are conceptually and methodologically difficult to estimate. Further, the mental health states at different stages of life also have to be seen. There is plenty of evidence that mental health problems are more pronounced in women for e.g. during the peri-natal period, particularly around birth, can affect the wellbeing, the psychological balance, and the attitudes of many mothers, making coping with the many tasks of child-care difficult [36] which is obviously absent or insignificant in men. The higher existences in the sample may be due to the fact that above factors are not prevalent in the studied sample.

These are more likely reciprocal associations rather than causally linked, which has not been much researched. In addition, data are emerging on the disproportionately high rates of suicide in the peri-natal period [37] for women. Fortunately, research has shown that with some help and support, most mothers can positively modify any difficulties they may be having with thinking, behaving, and caring for their 
babies [38]. This may improve, not only their own mental well being, but also provides better conditions for the optimal development of their babies. Once a mental health problem has been recognized in a woman, there are a series of community-based interventions that have demonstrated their usefulness and efficacy. These range from empathy and active listening; utilization of different psychosocial approaches and use of medication according to the woman's need. Methods applied will also depend on the severity of the condition, the ability and knowledge of health workers, and the local health and social infrastructure [39]. Overall a comprehensive consideration of mental health problems in women on various life stages cannot be ignored [30]. Therefore a concerted and articulated action is needed on the basis of gender. A vast literature also indicates the systematic changes - cultural, political and social - that are needed to alter well-entrenched patterns of violence, especially violence against women [40]. Violence linked to discrimination has its roots in social-structural relationships with a power imbalance between groups being the dominant feature. Thus, a range of strategies designed to address institutionalized violence is required. Health related hazards were found to be in increased levels on male subjects [18] a possible factor causing diminished mental health. Studies reveal that men were more vulnerable to the negative effects and to the negative outcomes of health. This conveys that the principles of equality and benevolence have some attribute over mental health which is in line with the results. In the present sample hailed from Indian culture still majority of the women take up the secondary role in the family system. Due to which they gain an inherent cushion effect which protects them and enables them to sustain and maintain effective mental health. Even with the advent of westernized practices and adaptations, the traditional roots of our culture which puts more emphasis on religious activities towards women are evident inherently from the results. Studies show that women have more health values than men combined with formal education is positively related to valuing spirituality [41].

\section{CONCLUSIONS}

Gender-blind studies fail to account for disparate life trajectories that are influenced by interactions among genetic endowment, environmental exposure and social and political environments. The lives of women and men, the relationships that they establish and their work, have changed dramatically in the past 50 years. Gender roles have changed radically. Researchers have found that empirically derived principles better matched with today's realities of gender, work and family rather than old theories. The apparent lack of progress in mainstreaming gender in health may be attributed to increasing privatization and retraction of the state's role in health [42]. Changes on mental health induced by religion were independent of age only when the people become older [43]. The difference between paid and unpaid work especially by women [44] in household work makes a difference. A study from the Philippines showed that women who engaged in paid work improved the quality of their diets [45]. In a clinical perspective also there are added advantages with the study of Gender. Certain features of disturbed mental health, such as social withdrawal, anxiety, attention problems and delinquency, are strong predictors of depression. These are liable for breaking down of an individual. Studies reveals suicidal ideation can be predicted by gender (female), amount of anxiety/depression, and delinquent problems [46].

No research is an end by itself. It is a continuous process which helps in the development of the science. A lot of issues still need to be understood in the area of mental health. International bodies need to ensure that countries at all stages of economic development are aware of the importance of mental health to community development. They can also encourage them to assess the possibilities and evidence for mental health of their population. However the scope and outcomes of Mental Health promotion activities are potentially wide and difficult to grasp.

\section{REFERENCES}

1. Doyal L. Sex, Gender and the 10/90 Gap in Health Research. Geneva: Global Forum on Health ; 2002.

2. Thomas S, Corinne D, Natacha N. A Comprehensive Analysis: Chronic diseases in Canadian Population: Public Health agency of Canada World Health Organization; 2000.

3. World Health Organization (WHO). The world health report 2001. New understanding, new hope. Geneva, (C) WHO; 2001. 
4. Greaves L. CIHR 2000: Sex, Gender and Women's Health. Vancouver: BC Centre of Excellence for Women's Health ; 2000.

5. Sharma S, Sharma M. Preventing Mental Illness and Promoting Health and Well being. Psychological Studies 2007; 52(1):70-6.

6. $\quad$ Freud S. Psychoanalytical theory of Development. Basic Books: UK; 1916.

7. Lahtinen E. Framework for promoting mental health in Europe. Helsinki, National Research and Development Centre for Welfare and Health (STAKES) ; 1999.

8. McCullough ME, Hoyt WT, Larson DB, Koenig HG, Thoresen C. Religious involvement and mortality: a meta-analytic review. Health Psychol 2000;19(3):211-8.

9. Syme L. To prevent disease: the need for a new approach. In: Blane D, Brunner E, Wilkinson R, eds. Health and social organisation. London, Routledge; 1996.

10. World Health Organization (WHO) Health Report. The world health report 2008: primary health care now more than ever. WHO ; 2008.

11. Whitehead M, Dahlgren G. Concepts and principles for tackling social inequities in health: Levelling up part 1. Copenhagen, World Health Organization Regional Office for Europe, 2008.

12. Starfield B, Shi L. Policy relevant determinants of health: an international perspective. Health Policy 2002;60:201-18.

13. Marmot M, Ryff CD, Bumpass LL, Shipley M, Marks NF. Social inequalities in health: next questions and converging evidence. Soc Sci Med 1997;44(6):901-10.

14. Shevlin M, Houston JE, Dorahy MJ, Adamson G. Cumulative traumas and psychosis: An analysis of the National Comorbidity Survey and the British Psychiatric Morbidity Survey. Schizo Bull 2008;34(1):193-9.

15. Sarafino E. Health Psychology (2nd ed.). New York: John Wiley \& Sons, Inc ; 1993.

16. Helen H, Shekhar S \& Moodie R. Promoting Mental Health Concepts. Emerging evidence and practice. A Report of the World Health Organization, Department of Mental Health and Substance Abuse in collaboration with the Victorian Health Promotion Foundation and The University of Melbourne; 2005.

17. Cochran SD, Mays VM. Relation between psychiatric patients \& adolescents. J Clin Psychol 2000;62:228-42.

18. Finch BK, Kolody B, Vega WA. Perceived discrimination and depression among Mexican-origin adults in California. J Health Soc Behav 2000;41(3):295-313.

19. Chemali J, Shipley MJ, Fuhrer R,Stansfeld SA. Gender, social relations and mental health: prospective findings from an occupational cohort. J Soc Sci Med 1999;48(1):77-87.

20. Harrison K. The importance of the educated healthy woman in Africa. Lancet 1997;349:644-7.

21. Sinha K. Methodology for understanding Indian culture. Copenhagen Journal of Asian Studies 2004;19(1):89-104.

22. Veena $M$, Khadi PB. Influence off family functioning on adolescent adjustment. Asian $\mathrm{J}$ Psychol Educ2004;37(5-6):2-7.

23. Newman BM, Newman RP. Development through life .A psychosocial approach. Sixth Ed Brooke/Cole Publishing Company; 1995.

24. Dolbier CL, Steinhardt MA. The development and validation of the Sense of Support Scale. Behav Med 2000;25(4):169-79.

25. Snow RC. Sex, gender and vulnerability. Glob Pub Health 2008;3(Suppl 1):58-74.

26. Courtney W. Engendering Health: A Social Constructionist Examination of Men's Health Beliefs and Behaviors. Psychol Men Mascuinity 2000;1(1):4-15.

27. Neubert MJ, Palmer LD. Emergence of women in healthcare leadership: transforming the impact of gender differences. J Mens Health Gender 2004;1(4):383-7.

28. Mayhew C. Occupational violence: a neglected occupational health and safety issue? Pol Pract Health Safe 2003;1(1):31-58.

29. Arcand R, Labrèche F, Messing K, Stock S, Tissot F. Environnement detravail et santé. In: Enquête sociale et de santé. Québec : Institut de lastatistique du Québec ; 2000. pp 525-70.

30. Aiken L, Clarke SP, Sloane DM, Sochalski J, Silber JH. Hospital nurse staffing and patient mortality, nurse burnout, and job dissatisfaction. JAMA 2002;288:1987-93.

31. George A. Nurses, community health workers, and home carers: gendered human resources compensating for skewed health systems. Glob Pub Heath 2008;3(Suppl 1):75-89.

32. Adams O. Life Expectancy in Canada-An Overview. Health Rep 1990;2(4):361-76.

33. Tytherleigh MY, Jacobs PA, Webb C, Ricketts C, Cooper C. Gender, Health and Stress in English University Staff-Exposure or Vulnerability. Applied Psychol 2007;56(2):267-87.

34. Singh AK. Tests Measurements, and Research Methods in Behavioral Sciences. Bharathi Bhawan Publishers and Distributors New Delhi; 2005.

35. Artazcoz L, Borrell C, Benach J. Gender Inequalities in Health among Workers: And Family Demands. J Epidemiol Commun Health 2007;55:639-47.

36. Wilkins R, Adams OB. Health expectancy in Canada, late 1970s: demographic, regional, and social dimensions. Am J Pub Health 1983;73(9):1073-80.

37. Goodman JH. Paternal postpartum depression, its relationship to maternal postpartum depression, and implications for family health. J Adv Nurs 2004;45(1):26-35.

38. Dayan J, Creveuil C, Herlicoviez M, Herbel C, Baranger E, Savoye C, Thouin A. Role of anxiety and depression in the onset of spontaneous preterm labor. Am J Epidemiol 2002;155(4):293-301. 
39. Rychetnik L, Todd A. Promoting mental health and well-being. An evidence review conducted for Vic Health. Melbourne, Victorian Health Promotion Foundation; 2004.

40. Miller WR, Thoresen CE. Spirituality, religion, and health. An emerging research field. Amer Psychol 2003;58:24-35.

41. Ravindran TKS, Kelkar-Khambete A. Gender mainstreaming in health: looking back, looking forward. Glob Pub Health 2008;3(Supp1 1):121-42.

42. Hays JC, Landerman LR, Blazer DG, Koenig HG, Carroll JW, Musick MA. Aging, health and the electronic church. J Aging Health 1998;10(4):458-82.

43. Brisson C, Laflamme N, Moisan J, Milot A, Masse B, Vezina M. Effect of family responsibilities and job strain on ambulatory blood pressure among white-collar women. Psychosom Med 1999;61:205-13.

44. Bisgrove EZ, Popkin BM. Does women's work improve their nutrition: evidence from the urban Philippines. Soc Sci Med 2004;40:1475-88.

Acknowledgements - Nil;

Conflict of Interest - Nil;

Funding - Nil. 